\title{
Clinical application of invalid foods using mealworms and evaluation of nutrition status and immune function: a study protocol for a randomized, double blind, placebo-controlled trial
}

Hyung Sun Kim', Yun Sun Lee ${ }^{1}$, Soo Yun Jang ${ }^{2}$, So Young Jun ${ }^{1}$, Jin Hong Lim¹', Im Kyung Kim³, Hyung Mi Kim ${ }^{2}$ and Joon Seong Park ${ }^{1 *}$

\begin{abstract}
Background: Protein intake is important for the recovery of the immune system, physical strength, and wound healing after surgery. Sarcopenia is associated with a poor prognosis when compared to patients without sarcopenia in cancer patients. Recently, edible insects, such as mealworms, have been recognized as having a high protein content. In this study, we will evaluate the effect of nutritional status and immune function change based on a patient's ingestion of mealworms after hepatobiliary pancreatic surgery.

Methods/design: This is a prospective, two-armed, phase III study investigating the effect of mealworm improving nutrition and immune status in patients after hepatobiliary pancreatic surgery. In the trial group, the patients will be provided with mealworms for 2 months after surgery. In the control group, patients will be provided with grain powder for 2 months after surgery. The target for accrual is 168 patients. We divided in to three groups according to the type of surgery.

Discussion: The primary endpoint is to evaluate body cell mass index 2 months postoperatively. Secondary endpoints include other body composition changes as well as nutrition index and immune function change. We expect that ingestion of mealworms can effectively improve the nutritional status and enhance the immune function. Mealworm can be used effectively for nutritional management of patients after surgery.
\end{abstract}

Trial registration: Clinicaltrials.gov NCT03201926 Registered June 28, 2017, retrospectively registered.

Keywords: Nutritional balance, Malnutrition, Immune function, Invalid foods

\section{Background}

Approximately $20-50 \%$ of hospitalized patients are reported to be malnourished [1]. The nutritional status of a patient generally declines during hospitalization $[2,3]$. Poor food intake during hospitalization is caused by deterioration of nutritional status, and malnutrition is often associated with complications including infection,

\footnotetext{
* Correspondence: JSPARK330@yuhs.ac

'Pancreatobiliary Cancer Clinic, Department of Surgery, Gangnam Severance Hospital, Yonsei University College of Medicine, 20, Eonju-ro 63-gil, Gangnam-gu, Seoul 06229, Korea

Full list of author information is available at the end of the article
}

increased number of hospital days, and increased mortality $[1,4,5]$. The American Society of Parenteral and Enteral Nutrition guidelines suggests that patients at risk of malnutrition should be provided with over $80 \%$ of their calorie and protein requirements within 2 to 3 days [6]. Patients with sarcopenia imbalance generally have a lower 5-year survival rate in cancer patients [7]. The quality of protein intake is important for the recovery of the immune system, physical strength, and wound healing after surgery; this is problematic since the patient's protein intake is generally quite poor [8].

(c) The Author(s). 2019 Open Access This article is distributed under the terms of the Creative Commons Attribution 4.0 International License (http://creativecommons.org/licenses/by/4.0/), which permits unrestricted use, distribution, and 
In particular, patients who undergo a pancreatectomy or hepatectomy usually have a severe imbalance in their nutritional status due to the disruption of digestive function and nutritional absorption postoperatively. Therefore, it is necessary to use high-quality protein-derived foods that can effectively increase protein intake. The United Nations Food and Agriculture Organization (FAO) is actively encouraging food production of edible insects as a new food source for the future. Currently, various insects are used in food in many regions, including Africa, Asia, South America, and Australia. The edible insects are high in protein content (50-60\%) and contain a large amount of fat, fiber, vitamins, and minerals. Tenebrio molitor (mealworm), which is an edible insect, contains a large amount of protein and unsaturated fatty acid. The nutritional components of these mealworm are thought to be of value as nutrient sources of high quality in a small amount of patients who need high nutrition.

The purpose of this study is to evaluate the development of long-term personalized products using mealworm and the enhancement of stability, nutritional status and immune status according to ingestion.

\section{Methods/design}

\section{Study design and period}

The trial is a single center, two-armed phase III study. The trial has been registered at www.clinicaltrials.gov (NCT03201926). Patients will be recruited by the Pancreatobiliary Cancer Clinic, Gangnam Severance Hospital, Yonsei University College of Medicine. The expected total duration of patient accrual is 2 years and 6 months and the follow-up period is 6 months.

\section{Study objectives and endpoints}

The primary endpoint is to evaluate body cell mass index at 2 months postoperatively. Secondary endpoints include other body composition changes as well as nutrition index and immune function.

\section{Patient selection and enrollment criteria Inclusion criteria}

- Patients scheduled for surgery with pancreatobiliary disease and liver cancer (HCC, CCC, and metastatic liver cancer)

- Karnofsky performance status $\geq 70$

- Patients who provide informed consent

\section{Exclusion criteria}

- Patients who underwent palliative surgery

- Patients with uncontrolled preoperative conditions

- Previous history of surgery affecting nutritional status (ex, gastrectomy, colectomy, etc)
- Pregnant and lactating women

- Patients with an allergy to mealworms

\section{Sample size calculation}

The output of the sample size will be based on an independent two-sample $t$ test. It is expected that there will be a difference in body cell mass between the standard meal group (control) and the group ingesting the mealworms. Assuming an alpha value of 0.05 and $1-\beta$ (power) of 0.8 , 75 samples will need to be obtained for each group; considering the dropout rate of $10 \%, 84$ samples will need to be obtained for each group. The total of the two groups is 168. The primary endpoint will be the number of subjects and body cell mass. A comparison will then be made between the two groups at 2 months. A subgroup analysis of pancreatobiliary and liver disease will also be performed as a secondary endpoint. Patients with pancreatobiliary and liver disease in our clinic have a 2:1 ratio. Therefore, the 168 patients were divided into two groups: one with 112 patients and the other with 56 patients. Patients with pancreaticobiliary disease (Group A \& B) and liver (Group C) disease were assigned through stratified randomization. Pancreatobiliary disease group is divided into enteral feeding group(Group A) and non enteral feeding group.(Group B).

\section{Pretreatment evaluation}

All patients who are potential candidates for hepatopancreatobiliary surgery will undergo a standard evaluation that will include contrast-enhanced computed tomography (CT), endoluminal ultrasound (EUS), magnetic resonance imaging (MRI), and positron emission tomography (PET-CT), which will be discussed at the preoperative conference.

Patients recommended for major hepatopancreatobiliary surgery (such as a liver resection, pancreaticoduodenectomy, or total/distal pancreatectomy) will be contacted and provided with a participant information sheet. Patients will be divided into three groups according to the type of surgery.

\section{Treatment \\ Trial intervention}

Patients receiving dietary supplementation with mealworms after their pancreatectomy, bile duct resection and hepatectomy will be given the mealworms during their hospitalization period and for another 2 months after discharge. The patients in group A will undergo enteral feeding after surgery (for those who undergo a pancreaticoduodenectomy, nutrients will be administered by an enteral feeding tube for early feeding while securing the pancreatojejunostomy). A feeding nasojejunal tube will be placed for patients in the enteral feeding group. Enteral feeding (Newcare 300 RTH, Daesang, 
Korea) will start within $24 \mathrm{~h}$ postoperatively at a rate of $20 \mathrm{~mL} / \mathrm{h}$. The velocity will be progressively increased by $20 \mathrm{~mL} / \mathrm{d}$ until reaching the full nutritional goal $(25 \mathrm{kcal} /$ $\mathrm{kg})$. Enteral feeding will be delivered by an infusion pump for $18 \mathrm{~h} /$ day with $6 \mathrm{~h}$ of rest.

On postoperative day 7 , abdominal and pelvic CT scans will be obtained, and if there are no complications, we will then start patients on a clear liquid diet for 1 day. In addition, the patients will be permitted to have a full liquid diet for 2 days and then a soft diet for 3 days following the liquid diet.

The patients of group B will undergo oral feeding 2 days after the surgery. (distal pancreatectomy, total pancreatectomy and bile duct resection) We will start patient's clear liquid diet for 1 day. The patients will be permitted to have a full liquid diet for 2 days and soft diet for 3 days following the liquid diet.

The patients of group $\mathrm{C}$ will undergo oral feeding 2 days after surgery (which includes liver resections). We will then start the patients on a clear liquid diet for 1 day. In addition, the patients will be permitted to have a full liquid diet for 2 days and soft diet for 3 days following the liquid diet. The mealworm powder include $3 \mathrm{~g}$ of carbohydrate, $14.4 \mathrm{~g}$ of protein and $11 \mathrm{~g}$ of fat, $163 \mathrm{kcal}$ per $30 \mathrm{~g}$. (Table 1) As a result of a nutritional analysis of mealworm powder, $10.28 \mathrm{~g}$ of carbohydrate, $48.26 \mathrm{~g}$ of protein and $35.81 \mathrm{~g}$ of fat were found per $100 \mathrm{~g}$. In particular, the protein includes essential amino acids as well as many unsaturated fatty acids ranging from 76 to $80 \%$ of the total fatty acids. In addition, iron and calcium and other minerals were found (Table 2).

\section{Control intervention}

Patients receiving dietary supplementation with grainpowder after pancreatectomy and hepatectomy will be given grainpowder for their hospitalization period and 2 months after discharge. The grainpowder include $23 \mathrm{~g}$ of carbohydrate, $2.9 \mathrm{~g}$ of protein and $0.5 \mathrm{~g}$ of fat, $106 \mathrm{kcal}$ per $30 \mathrm{~g}$. Group A will receive the grainpowder on postoperative day 7 . And group $B$ and $C$ will receive grainpowder in the same dose as trial group's mealworms at the start of diet at postoperative 2 days (Table 3 ).

\section{Outcome measures}

The primary outcome is the body cell mass index as measured by body composition (Inbody S-10 (Biospace, Seoul, Korea)). The secondary outcome measures are as follows: nutritional index (weight, soft lean mass, fat free mass, fat mass, PG-SGA [Scored Patient-Generated Subjective Global Assessment]) and immune function test (that assesses $\mathrm{T}$ cells, B cells, cytokines). We will confirm changes in immune cells through FACS (fluorescence-activated cell sorting). Based on the immune assay using blood samples from patients, we will identify changes in several cytokines.

\section{Timeline and recruitment \\ Recruitment of study participants}

Once the patients' surgery is confirmed, the research staff will give the study staff a list of the patients. After determining the suitability of the patient, the study staff will assign the patient to the test or control group in a randomized sequence in the order in which they are enrolled. In addition, they will be assigned in a double-blinded manner.

\section{Randomization}

The researchers will select patients after obtaining consent. Randomization will take place via an allocation randomization system 2 days before the surgery, which will be directed by our department. Patients will be randomized to one of the treatment groups in a 1:1 ratio. After consent for study enrollment is obtained, the randomization process will be applied to identify the next allotment. The surgeons will be blind to the allotment throughout the enrollment process.

\section{Blinding}

Patients and all team members will be blinded to the intervention. Adverse event (AE) and serious adverse event (SAE) need to be reported as soon as they are noted. This will not have any impact on the endpoint assessment of the patient.

Table 1 Components of the Trial group's diet

\begin{tabular}{|c|c|c|c|}
\hline & Mealworm & Protein $(\mathrm{g})$ & Supplement \\
\hline Step 1 diet (Clear liquid diet) & $10 \mathrm{~g}(1$ piece $)$ & $5.34 \mathrm{~g}$ & Mealworm 1 piece \\
\hline Step 2 diet (Full liquid diet) & $30 \mathrm{~g}(3$ pieces $)$ & $14.4 \mathrm{~g}$ & Mealworm 3 pieces \\
\hline Step 3 diet (Soft diet) & $40 \mathrm{~g}(4$ pieces) & $21 \mathrm{~g}$ & + Mealworm 3 pieces \\
\hline
\end{tabular}


Table 2 Menu plan and protocol of the hospital meals for the mealworm and control groups

\begin{tabular}{|c|c|c|c|c|}
\hline & & & Mealworm group & Control group \\
\hline Step 1 & $\begin{array}{l}\text { Menu } \\
\text { Nutrient }\end{array}$ & $\begin{array}{l}\text { Energy } \\
\text { Protein } \\
\text { Carbohydrate } \\
\text { Fat }\end{array}$ & $\begin{array}{l}\text { Carbohydrate-based liquid diet, } \\
\text { Mealworm jelly } \\
580 \mathrm{kcal} \\
13 \mathrm{~g} \\
114 \mathrm{~g} \\
8 \mathrm{~g}\end{array}$ & $\begin{array}{l}\text { Carbohydrate-based liquid diet, } \\
\text { Juice } \\
450 \mathrm{kcal} \\
16 \mathrm{~g} \\
84 \mathrm{~g} \\
6 \mathrm{~g}\end{array}$ \\
\hline Step 2 & $\begin{array}{l}\text { Menu } \\
\text { Nutrient }\end{array}$ & $\begin{array}{l}\text { Energy } \\
\text { Protein } \\
\text { Carbohydrate } \\
\text { Fat }\end{array}$ & $\begin{array}{l}\text { Mealworm soup, Mealworm shake } \\
\text { with berry, } \\
\text { Protein-fortified gelatin } \\
1220 \mathrm{kcal} \\
50 \mathrm{~g} \\
180 \mathrm{~g} \\
34 \mathrm{~g}\end{array}$ & $\begin{array}{l}\text { Carbohydrate-based liquid diet, } \\
\text { Thin low-fat soup, } \\
\text { Soybean milk, Protein-fortified } \\
\text { gelatin } \\
1240 \mathrm{kcal} \\
43 \mathrm{~g} \\
180 \mathrm{~g} \\
39 \mathrm{~g}\end{array}$ \\
\hline Step 3 & $\begin{array}{l}\text { Menu } \\
\text { Nutrient }\end{array}$ & $\begin{array}{l}\text { Energy } \\
\text { Protein } \\
\text { Carbohydrate } \\
\text { Fat }\end{array}$ & $\begin{array}{l}\text { Deluxe rice porridge with soft side } \\
\text { dishes, } \\
\text { ONS1)-based mealworm drink, } \\
\text { Mealworm tea-confectionery } \\
1629 \mathrm{kcal} \\
81 \mathrm{~g} \\
225 \mathrm{~g} \\
45 \mathrm{~g}\end{array}$ & $\begin{array}{l}\text { Rice porridge with soft side dishes } \\
1600 \mathrm{kcal} \\
75 \mathrm{~g} \\
235 \mathrm{~g} \\
40 \mathrm{~g}\end{array}$ \\
\hline Protocol & & & \multicolumn{2}{|c|}{$\begin{array}{l}\text { The doctor prescribes Step } 1 \text { diet (clear liquid diet) as the first meal after surgery. Patients eat this diet for } 2-3 \\
\text { meals. As the next meal, the doctor prescribes Step } 2 \text { diet (full liquid diet). Patients eat this diet for } 2-3 \text { meals. If } \\
\text { the diet transition goes smoothly, the doctor prescribes Step } 3 \text { diet (soft diet). Patients eat this diet until } \\
\text { discharge. The doctor prescribes hospital meal intake. Depending on the patient's condition, the steps for } \\
\text { consuming the diet could be adjusted. The dietitian surveys the dietary intake every day until discharge. If there is } \\
\text { a meal-related side effect, it is reported to the doctor. }\end{array}$} \\
\hline
\end{tabular}

\section{Data management}

The amount of food consumed by the patient daily will be assessed as a diary entry. The researcher collects the bags left over from the patients at the outpatient clinic. Individual participant medical information obtained as a result of this study is considered confidential and disclosure to third parties is prohibited. Blood samples transferred to the laboratory of Gangnam Severance Hospital will be identifiable by unique trial numbers only. Results related to nutrient indicators (Inbody, PG-SGA) will be managed by a Gangnam Severance Nutrition Team. The blood sample associated with the immune function test will be transferred to the lab of the study staff in Gangnam Severance Hospital, which will then undergo FACS (Fluorescenceactivated cell sorting).

\section{Withdrawal and reporting of adverse effects}

If patients wish to voluntarily withdraw from the study, the patient will be asked if they would be given medical care until any AE symptoms resolve or the patient's condition becomes stable.

Patients can quit the trial at any point without the need to give reasons for their decisions. If voluntary withdrawal occurs prior to diet intervention, the patient will not be randomized, and no more trial data will be collected for that patient. Patients can also withdraw from the trial following diet intervention.

\section{Statistical methodology}

All data will be entered into a single Microsoft Excel spreadsheet with participants identified only by their

Table 3 Components of the Control group's diet

\begin{tabular}{|c|c|c|c|}
\hline & Grainpowder & Protein $(\mathrm{g})$ & Supplement \\
\hline Step 1 diet (Clear liquid diet) & $10 \mathrm{~g}(1 \mathrm{piece})$ & $1.0 \mathrm{~g}$ & Grainpowder 1 piece \\
\hline Step 2 diet (Full liquid diet) & $30 \mathrm{~g}(3$ pieces $)$ & $2.9 \mathrm{~g}$ & Grainpowder 3 pieces \\
\hline Step 3 diet (Soft diet) & $40 \mathrm{~g}(4$ pieces) & $4.8 \mathrm{~g}$ & Grainpowder 3 pieces \\
& & & Grainpowder jelly 3 pieces \\
\hline
\end{tabular}


unique subject number. All entries will be double-checked by another member of the research team. Statistical analysis will be performed using IBM SPSS version 21.0 (IBM Corp; released 2012. IBM SPSS Statistics for Windows Version 21.0. Armonk, NY, USA). The mean difference in nutrient intakes, changes in body measurements, changes in body composition, and PG-SGA scores will be calculated using the Wilcoxon signed rank test and the Mann Whitney test.

\section{Monitoring and follow-up period}

The research team is responsible for monitoring the study. At 1 week after discharge, the participants are asked whether they are willing to continue ingesting the mealworm. Every 2 weeks after the patients visit their outpatient clinic, the monitoring researcher will check whether they are taking the mealworm regularly. The research team is also responsible for systematically reviewing the causes of withdrawal from the study. Assessment of these changes will be determined 2 months after surgery (Table 4).

\section{Ethical and legal considerations}

The study protocol was approved by the institutional review board at Gangnam Severance Hospital, Yonsei University of Korea (3-2017-0077). The study complies with the Declaration of Helsinki and the principles of Good Clinical Practice.

Table 4 Clinical trial timeline

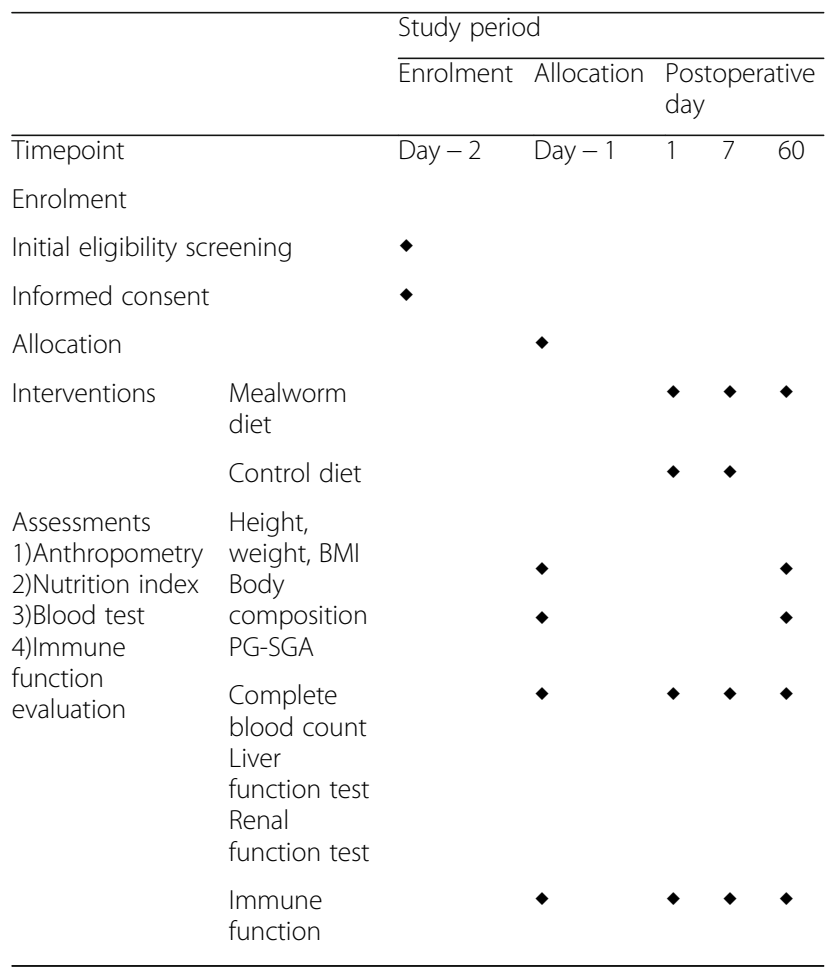

\section{Discussion}

The aim of this study is to evaluate the effectiveness and improvement of nutritional status and immune function based on the intake of invalid foods in postoperative patients for major surgery (such surgery of the pancreas, bile duct and liver) after an intervention period of 2 months. Nutritional deficiencies are frequent in patients after abdominal surgery. Malnutrition is an important risk factor for developing sarcopenia [9-12]. The decrease in the amount of fat free mass is associated with an increased mortality rate in patients at risk of malnutrition such as surgery and critical illness [13]. Protein-enhanced diets are known to increase survival in these patients, and therefore, nutritional support using high-protein foods is important [14]. Based on studies such as these, high protein foods using mealworm are now being used. However, only a few studies have evaluated the enhancement of immune function in patients after intake of high protein foods. In other studies, immune function evaluations were reported $[15,16]$. Early enteral nutrition after esophageal cancer surgery can effectively improve the nutritional status and enhance the immune function [15] . Some markers of immune function may be useful for distinguishing patients with good or bad prognoses after head and neck cancer surgery by checking the cytokine levels [16] . However, there are no studies on the effects of long term use $(2$ months) of high protein mealworm that can be used effectively for nutritional management of patients after surgery. This will be the first study to directly identify the relationship between nutritional status and immune function after surgery.

\section{Abbreviations}

AE: Adverse Event; CT: Computed Tomography; EUS: Endoscopic Ultrasound; FACS: Fluorescence-Activated Cell Sorting; MRI: Magnetic Resonance Imaging; PET-CT: Positron Emission Tomography; PG-SGA: Scored PatientGenerated Subjective Global Assessment; SAE: Serious Adverse Event

\section{Acknowledgements}

Pancreatobiliary Cancer Clinic, Department of Surgery, Gangnam Severance Hospital, Yonsei University, Seoul, Korea.

Department of Nutrition and Dietetics, Gangnam Severance Hospital, Yonsei University, Seoul, Korea.

Authors' contributions

Conceptualization, Supervisor: JSP Coordinated recruitment, participant management: HMK, JHL, SYJang, SYJun, IKK, YSL, JSP. Formal analysis: HSK, SYJang, JSP. Methodology: HSK, YSL, JSP. Writing - original draft: HSK. Writing - review \& editing: JSP. All authors read and approved the final manuscript.

\section{Funding}

This paper presents independent research funded by the support of 'cooperative Research Program for Agriculture Science and Technology Development (Project No: PJ 012802022018)' Rural Development Administration, Republic of Korea. The Rural Development Administation has no role in study desing;collection, management, analysis, and interpretation of data; writing of the report; or the decision to submit the report for publication. 


\section{Availability of data and materials}

Data sharing is not applicable to this article as no datasets have been generated or analysed for this study yet.

\section{Ethics approval and consent to participate}

The study protocol was approved by the institutional review board at Gangnam Severance Hospital, Yonsei University of Korea (3-2017-0077) Participants will provide written informed consent for participation.

\section{Consent for publication}

Not Applicable.

\section{Competing interests}

The authors declare that they have no competing interests.

\section{Author details}

'Pancreatobiliary Cancer Clinic, Department of Surgery, Gangnam Severance Hospital, Yonsei University College of Medicine, 20, Eonju-ro 63-gil, Gangnam-gu, Seoul 06229, Korea. ${ }^{2}$ Department of Nutrition and Dietetics, Gangnam Severance Hospital, Yonsei University, Seoul, Korea. ${ }^{3}$ Department of Surgical critical care medicine, Severance Hospital, Yonsei University, Seoul, Korea.

Received: 27 June 2019 Accepted: 22 August 2019

Published online: 18 November 2019

\section{References}

1. Norman K, Pichard C, Lochs H, Pirlich M. Prognostic impact of diseaserelated malnutrition. Clin Nutr. 2008;27(1):5-15.

2. Bistrian BR, Blackburn GL, Hallowell $E$, Heddle R. Protein status of general surgical patients. JAMA. 1974;230(6):858-60.

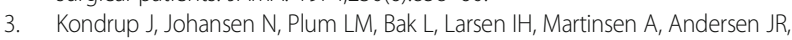

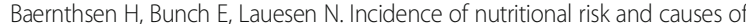
inadequate nutritional care in hospitals. Clin Nutr. 2002;21(6):461-8.

4. Allison SP. Malnutrition, disease, and outcome. Nutrition. 2000;16(7-8):590-3.

5. Sullivan DH, Sun S, Walls RC. Protein-energy undernutrition among elderly hospitalized patients - a prospective study. Jama-J Am Med Assoc. 1999. 281(21):2013-9.

6. McClave SA, Taylor BE, Martindale RG, Warren MM, Johnson DR, Braunschweig C, McCarthy MS, Davanos E, Rice TW, Cresci GA, et al. Guidelines for the provision and assessment of nutrition support therapy in the adult critically ill patient: Society of Critical Care Medicine (SCCM) and American Society for Parenteral and Enteral Nutrition (A.S.P.E.N.). JPEN J Parenter Enteral Nutr. 2016;40(2):159-211.

7. van Vugt $J L$, Levolger S, Coelen RJ, de Bruin RW, IJzermans JN. The impact of sarcopenia on survival and complications in surgical oncology: a review of the current literature. J Surg Oncol. 2015;112(6):681-2.

8. Ho Sun Lee KHS, Rha SY, Chung MJ, Si YS, Song SE, Ham HJ, Kim HM Changes in nutrient intake in patients at nutritional risk. J Korean Diet Assoc. 2014;20(4):285-95.

9. Agarwal E, Ferguson M, Banks M, Bauer J, Capra S, Isenring E. Nutritional status and dietary intake of acute care patients: results from the nutrition care day survey 2010. Clin Nutr. 2012;31(1):41-7.

10. Delmi M, Rapin $\mathrm{CH}$, Bengoa JM, Delmas PD, Vasey H, Bonjour JP. Dietary supplementation in elderly patients with fractured neck of the femur. Lancet. 1990;335(8696):1013-6.

11. Olin AO, Osterberg P, Hadell K, Armyr I, Jerstrom S, Ljungqvist O. Energyenriched hospital food to improve energy intake in elderly patients. JPEN J Parenter Enteral Nutr. 1996;20(2):93-7.

12. Hu X, Zhang L, Wang H, Hao Q, Dong B, Yang M. Malnutrition-sarcopenia syndrome predicts mortality in hospitalized older patients. Sci Rep. 2017 7(1):3171.

13. Thibault R, Makhlouf AM, Mulliez A, Gonzalez MC. Fat free mass at admission predicts 28 day mortality in intensive care unit patients : the international prospective observational study phase angle project. Intensive Care Med. 2016;42:1445-53.

14. Weijs PJ, Cynober L, DeLegge M, Kreymann G, Wernerman J, Wolfe RR. Proteins and amino acids are fundamental to optimal nutrition support in critically ill patients. Crit Care. 2014;18(6):591.

15. Yu GP, Chen GQ, Huang B, Shao WL, Zeng GQ. Effect of early enteral nutrition on postoperative nutritional status and immune function in elderly patients with esophageal cancer or cardiac cancer. Chin J Cancer Res. 2013; 25(3):299-305.

16. van Bokhorst-de van der Schueren MAE, Quak JJ, von Blomber-van der Flier BME, Kuik DJ, Langendoen SI, Snow GB, Green CJ, van Leeuwen PAM. Effect of perioperative nutrition, with and without arginine supplementation, on nutritional status, immune function, postoperative morbidity, and survival in severely malnourished head and neck cancer patients. Am J Clin Nutr. 2001; 73(2):323-32.

\section{Publisher's Note}

Springer Nature remains neutral with regard to jurisdictional claims in published maps and institutional affiliations.
Ready to submit your research? Choose BMC and benefit from:

- fast, convenient online submission

- thorough peer review by experienced researchers in your field

- rapid publication on acceptance

- support for research data, including large and complex data types

- gold Open Access which fosters wider collaboration and increased citations

- maximum visibility for your research: over $100 \mathrm{M}$ website views per year

At $\mathrm{BMC}$, research is always in progress.

Learn more biomedcentral.com/submissions 